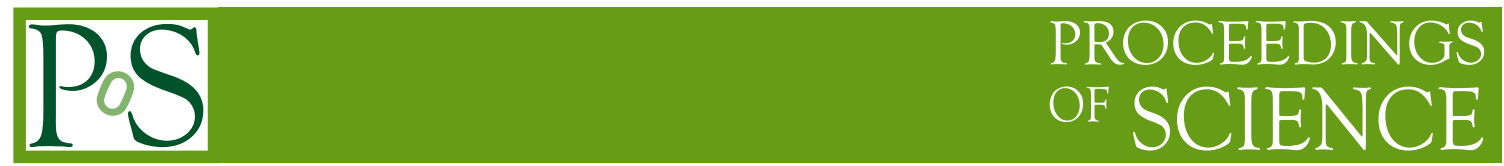

\title{
A quantum field theory for the atoms of space
}

\author{
Daniele Oriti* \\ Max Planck Institute for Gravitational Physics (Albert Einstein Institute) \\ E-mail: daniele.oriti@aei.mpg.de
}

\begin{abstract}
We give a brief introduction to the group field theory (GFT) formalism for quantum gravity, a 2nd quantised reformulation of loop quantum gravity and spin foam models and a group-theoretic enrichment of the purely combinatorial tensor models. We then review some recent key developments concerning: the definition and analysis of $4 \mathrm{~d}$ gravity models, progress in GFT renormalisation, and the extraction of effective cosmological dynamics from GFT condensates.
\end{abstract}

Frontiers of Fundamental Physics 14 - FFP14,

15-18 July 2014

Aix Marseille University (AMU) Saint-Charles Campus, Marseille

\footnotetext{
*Speaker.
} 